\title{
Tipografias em uso pelas universidades: um levantamento tipográfico a partir de websites universitários
}

\author{
Typographies in use by universities: a typographical survey on university websites
}

\author{
Susana Narimatsu Sato, Clice de Toledo Sanjar Mazzilli
}

tipografia digital, legibilidade, sites universitários, identidade visual, tipografia

O artigo discute o uso de fontes tipográficas em websites oficiais de instituições de ensino superior, com o propósito de identificar as práticas atuais de tipografia dentre sites universitários, e de forma a contribuir com o delineamento de parâmetros objetivos para o desenvolvimento de produtos eficientes em termos de legibilidade e de transmissão tanto dos conteúdos informativos quanto da identidade das instituições que representam. Para tanto, foram observados os sites das 15 universidades que obtiveram melhor avaliação na edição de 2020 do QS World University Rankings. Adotando metodologia de pesquisa documental, o levantamento reuniu 742 dados com o intuito inicial de identificar: as fontes mais frequentes em sites de universidades; se há predominância entre tipos com ou sem serifa; o corpo (tamanho) médio das fontes; a proporção média entre corpo e entrelinha; as combinações de cor de texto e cor de fundo mais frequentes; se há alguma relação entre características da universidade e as fontes selecionadas. Concluiu-se que os sites universitários têm acompanhado os grandes portais de notícias no tocante à aplicação dos tipos (corpo, entrelinha, alinhamento, hifenização e cores), mas não na seleção das fontes (havendo prevalência de tipos sem serifa nas páginas analisadas).

digital typography, readability, university websites, visual identity, typography

The paper discusses the use of typographic fonts on official university websites, in order to identify current typography practices among higher education institutions' websites, so as to contribute with objective parameters for the development of efficient products in terms of legibility, of transmitting the informative content and of communicating the institutions' identity. For this purpose, the websites of the 15 universities with the best evaluation in the 2020 edition of the QS World University Rankings were observed. Adopting a documental research methodology, 742 data were collected with the initial purpose of identifying: the most frequent typefaces on university websites; whether there is a predominance between types with or without serif; the average body height of the fonts; the average proportion between body height and leading; the most frequent text color and background color combinations; whether there is any relation between characteristics of the university and the selected fonts. It was concluded that university websites follow major news websites regarding the application of the types (body height, leading, alignment, hyphenation and color), but not in the selection of the typefaces (with a prevalence of sans serif fonts on the analyzed pages).

\section{Introdução}

A escolha e o modo de aplicação das fontes tipográficas em um texto ou conjunto de textos influencia a forma como os leitores percebem o conteúdo da mensagem (Unger, 2018, p. 175). Partindo do princípio de que uma seleção criteriosa dos tipos e decisões consistentes quanto à

Anais do $10^{\circ} \mathrm{CIDI}$ e $10^{\circ} \mathrm{CONGIC}$

Kelli C.A.S. Smythe, Rafael de Castro Andrade (orgs.)

Sociedade Brasileira de Design da Informação - SBDI

Curitiba | Brasil | 2021
Proceedings of the $10^{\text {th }} \mathrm{CIDI}$ and $10^{\text {th }}$ CONGIC

Kelli C.A.S. Smythe, Rafael de Castro Andrade (orgs.)

Sociedade Brasileira de Design da Informação - SBDI Curitiba | Brazil | 2021 
disposição dos textos favorecem tanto a facilidade e velocidade de leitura quanto a retenção da informação (Mozina et al, 2019, p. 172), o presente artigo pretende identificar o estado da arte, as práticas tipográficas atuais dentre websites oficiais de instituições de ensino superior, além de refletir sobre a eficiência das fontes empregadas em termos de legibilidade e de comunicação da identidade das instituições que representam.

Para tanto, foi realizado um levantamento de 742 dados acerca das fontes tipográficas utilizadas nos sites das 15 universidades que obtiveram melhor avaliação na edição de 2020 do QS World University Rankings (Tabela 1).

Publicado anualmente pela editora britânica Quacquarelli Symonds, o QS está entre as três principais classificações mundiais de universidades (Callaghan, 2017; Zirulnick, 2010), ao lado do Times Higher Education World University Rankings e do Academic Ranking of World Universities, também conhecido como Ranking de Xangai.

Tabela 1: Quinze universidades com melhores posições na edição de 2020 do QS World University Rankings. (Fonte: elaboração nossa com dados do QS World University Rankings e dos websites das respectivas universidades)

\begin{tabular}{llll}
\hline $\begin{array}{l}\text { Posição } \\
\text { no Ranking }\end{array}$ & Nome da Instituição & $\begin{array}{l}\text { Ano de } \\
\text { inauguração }\end{array}$ & Continente \\
\hline $1^{\circ}$ & MIT (Massachusetts Institute of Technology) & 1861 & América do Norte \\
$2^{\circ}$ & Stanford University & 1891 & América do Norte \\
$3^{\circ}$ & Harvard University & 1636 & América do Norte \\
$4^{\circ}$ & University of Oxford & 1096 & Europa \\
$5^{\circ}$ & Caltech (California Institute of Technology) & 1891 & América do Norte \\
$6^{\circ}$ & ETH Zurich & 1855 & Europa \\
$7^{\circ}$ & University of Cambridge & 1209 & Europa \\
$8^{\circ}$ & UCL (University College London) & 1826 & Europa \\
$9^{\circ}$ & Imperial College London & 1907 & Europa \\
$10^{\circ}$ & University of Chicago & 1890 & América do Norte \\
$11^{\circ}$ & NTU (Nanyang Technological University of Singapore) & 1991 & Ásia \\
$12^{\circ}$ & NUS (National University of Singapore) & 1905 & Ásia \\
$13^{\circ}$ & Princeton University & 1746 & América do Norte \\
$14^{\circ}$ & Cornell University & 1865 & América do Norte \\
$15^{\circ}$ & University of Pennsylvania & 1740 & América do Norte \\
\hline
\end{tabular}

\section{Metodologia}

Considerando de que uma boa tipografia define um web design efetivo, Constantin (2009) realizou uma pesquisa com mais de cinquenta sites de notícias relevantes no cenário 
internacional, concentrando-se nas escolhas e aplicações das fontes tipográficas. Foram analisadas páginas de jornais, revistas e outras publicações - a maioria de grande alcance, como o Boston Globe e o The Financial Times. Algumas publicações de menor abrangência foram também selecionadas por seu cunho bastante especializado e por atenderem a públicos mais exigentes - como no caso do A List Apart e do UX Booth.

Por se tratar de sites cujo conteúdo era primordialmente textual, entendeu-se que havia um planejamento criterioso para que esses conteúdos fossem apresentados de forma altamente legível, garantindo conforto na leitura e, consequentemente, promovendo a fidelidade do público. Para seu estudo, Constantin (2009) levantou dados objetivos, como o tamanho das fontes, a altura das entrelinhas, o espaço entre parágrafos, o comprimento ou a quantidade de caracteres por linha.

Quatro anos depois, Constantin (2013) repetiu a pesquisa mantendo o mesmo formato, o que permitiu a visualização de algumas transformações na prática tipográfica em sites de notícias neste ínterim.

Em apenas quatro anos, o cenário sofreu mudanças significativas. Constatou-se, por exemplo, que houve um aumento expressivo no uso de fontes com serifa em blocos de texto. Em 2009, 66\% das fontes de texto eram sem serifa, enquanto 34\% eram serifadas. Já em 2013 , houve uma inversão nos valores: apenas $36,5 \%$ das fontes de texto eram sem serifa, e $61,5 \%$ eram serifadas.

No caso das fontes de títulos, não chegou a haver uma inversão nas proporções, mas ainda assim as fontes com serifa ganharam espaço: Em 2009, 60\% das fontes de títulos eram sem serifa, enquanto $40 \%$ eram serifadas. Já em 2013 , 51\% eram sem serifa e $47,1 \%$ eram serifadas.

Reforçamos aqui que, embora tenha-se assumido por muito tempo que os tipos sem serifa sejam menos legíveis que os tipos com serifa, nenhuma evidência conclusiva foi encontrada (Unger, 2018, p. 171).

O corpo, isto é, a altura máxima do conjunto dos caracteres de uma fonte (Farias, 2016, p. 12), também sofreu um acréscimo nos quatro anos entre um estudo e outro. Enquanto em 2009 as fontes de títulos em telas grandes tinham em média 25,6 pixels, em 2013 esta dimensão havia escalado mais de 10 pixels, atingindo uma média de 38 pixels.

Quanto aos blocos de texto, Constantin (2013) acredita que foi em prol da legibilidade que as fontes foram aumentadas: de corpos majoritariamente entre 12 e 14 pixels em 2009, para 14 a 16 pixels em 2013. Isto condiz com a observação de Compton (2015) de que se no início da internet o tamanho padrão para tipos na tela costumava girar em torno dos 12 pixels, sendo possível encontrar tamanhos ainda mais reduzidos, atualmente "um número cada vez maior de designers está usando tipos maiores, a partir de 14 px, passando por 18 px e, até mesmo, chegando a 21 px" (Compton, 2015, p. 59).

De forma semelhante aos estudos de Constantin (2009; 2013), para o presente artigo foi também coletada uma série de dados a respeito dos tipos empregados nos sites das universidades em análise. Mas, diferentemente do estudo citado, pretendeu-se aqui verificar 
tanto questões objetivas - como, por exemplo, a razão entre o tamanho da fonte e a altura da entrelinha -, quanto de cunho mais semântico - como a relação entre o tempo de existência da universidade e o tradicionalismo na seleção de fontes; ou entre a localização geográfica e o tipo de fonte empregada. Alguns questionamentos que nortearam a pesquisa foram:

- Quais as fontes mais frequentes em sites de universidades?

- Existe uma predominância entre tipos com serifa ou sem serifa?

- Qual o tamanho/corpo médio das fontes?

- Qual a proporção média entre o corpo das fontes e suas respectivas entrelinhas?

- Quais as combinações de cor de texto e cor de fundo mais frequentes?

- Existe alguma relação entre a idade da universidade e o tradicionalismo das fontes empregadas?

- Existem tendências específicas em diferentes regiões geográficas?

Como mencionado anteriormente, foram observados os sites das universidades com as 15 melhores colocações na edição de 2020 do QS World University Rankings. Uma planilha com o levantamento completo, reunindo todos os 742 dados coletados para o presente trabalho, assim como alguns resultados, está disponível para visualização no seguinte link: https://bit.ly/3vPb9ap

Em cada site analisado, foram apuradas características de quatro grupos distintos de textos, a saber:

- Leads, i.e. as chamadas de notícias na página inicial do website

- Títulos nas páginas internas de notícia

- Blocos de texto nas páginas internas de notícia (geralmente os textos mais longos do site)

- Logo da universidade

Levantaram-se as seguintes características das fontes dentro de cada um dos quatro grupos acima citados:

1. Família da fonte

2. Corpo da fonte

3. Estilo da fonte (font style)

4. Peso da fonte (font weight)

5. Entrelinha

6. Alinhamento do texto

7. Hifenização do texto

8. Cor do texto

9. Cor do fundo

10. Classificação do tipo

11. Ano de publicação da fonte digital 
12. Ano do desenho original da fonte (no caso de versões digitais de fontes já existentes)

13. Inspiração para o desenho da fonte

14. Foundry/Designer

15. Se é uma fonte customizada

16. Se é uma fonte de distribuição gratuita

Tendo em vista que, em boa parte dos sites, o "Corpo da fonte" (2) costuma ser ajustado automaticamente conforme o tamanho da janela do navegador, todas as fontes deste levantamento foram observadas em uma janela de 1280 pixels de largura.

Para o item "Classificação do tipo" (10), utilizou-se o sistema de categorização simplificado proposto por Spiekermann e Ginger (1993, p. 50-51), que identificaram as fontes como: serif (com serifa), sans serif (sem serifa), script (escriturais), display (para títulos) ou symbols (símbolos).

No caso de características mais subjetivas, como a "Inspiração para o desenho da fonte" (13), as informações foram colhidas a partir de depoimentos publicados pelas respectivas type foundries ou pelos próprios designers das fontes. Na planilha que reúne os dados do presente levantamento, na coluna à direita de cada dado, está indicado o link da referência, isto é, o site ou a página de onde a informação foi coletada.

Um exemplo é a fonte Merriweather, empregada no site da Universidade de Harvard. Em seu blog, o designer da fonte, Eben Sorkin, afirma que ao projetá-la procurou manter o "sentimento encontrado" em tipos franceses do século XVI, como as fontes de Garamond, Jannon e Elzevir, bem como em tipos aldinos italianos do século XV. Sorkin segue descrevendo como buscou evocar um "sentimento familiar" de livros antigos (Sorkin, 2011, tradução nossa). Um dos recursos empregados foi o eixo de contraste inclinado (Figura 1). Segundo o designer, a maioria dos tipos contemporâneos projetados para tela emprega um eixo vertical por questões de praticidade: "o manejo do eixo inclinado é mais complicado e consome mais tempo que o eixo vertical em um projeto de tipo". Por outro lado, o eixo inclinado contribuiria para uma sensação de "tipo de livro antigo" (Sorkin, 2011, tradução nossa).

Figura 1: Comparação entre os eixos de contraste das fontes Droid Serif e Merriweather. (Fonte: Sorkin, 2011)

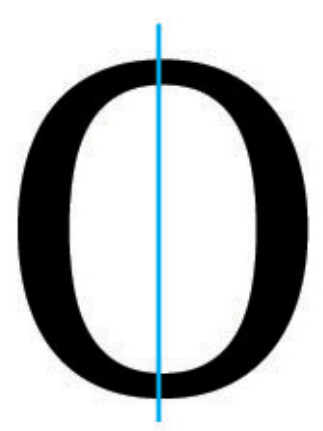

Droid Serif

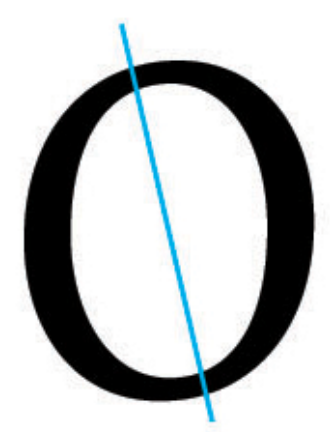

Merriweather 
Adicionalmente, foram também coletadas as seguintes informações acerca das universidades em questão:

- Ano de fundação da universidade

- Idade da universidade

- Continente em que a universidade está localizada

\section{Resultados}

Nos sites das 15 universidades analisadas, as fontes de texto nas notícias, isto é, os blocos de texto mais longos, têm em média corpos de 16,6 pixels. Isto está em concordância com o proposto por Compton (2015, p. 59), ao defender que, em telas, o tamanho de tipo ideal seja a partir de 14 pixels. O próprio autor, no entanto, aponta que para a fonte Georgia, por exemplo, um bom ponto de partida para visualização na tela seria em torno de 22,6 pixels. O corpo mínimo ideal, portanto, varia tanto conforme a fonte quanto as condições de leitura:

\footnotetext{
"Um tamanho que parece ideal na impressão provavelmente parecerá pequeno demais na tela de um computador de mesa ou de um laptop. As pessoas tendem a segurar livros e revistas relativamente próximos de seus rostos, enquanto sentam mais longe das telas de computadores." [Por outro lado] "em dispositivos móveis, os tipos podem ser menores do que em um computador de mesa, porque o usuário pode ajustar a distância entre a tela e seu rosto para obter o tamanho mais adequado." (Compton, 2015, p. 58-59)
}

Quanto aos títulos das notícias nos sites analisados, o corpo médio é de 43,5 pixels, sendo o menor corpo encontrado de 25 pixels e o maior de 78,2 pixels. Variação maior foi verificada entre os tamanhos dos tipos nos leads, ou seja, nas chamadas das home pages: o menor tinha 16 pixels e o maior 54,9 pixels, chegando-se a uma média de 30,7 pixels.

Nos blocos de texto das notícias, a razão média entre as entrelinhas e o corpo das fontes é 1,517. Tal valor está bastante próximo da proporção de 1,5, frequentemente recomendada na literatura sobre tipografia (Constantin, 2009).

Reforçamos aqui que em cada site foram analisados, além da fonte do logo da universidade, três grupos de texto, a saber: leads, títulos de notícias e textos de notícias. Desta forma, à parte dos logotipos, foram analisados nos 15 sites um total de 45 conjuntos de textos.

Destes 45 conjuntos, $82,2 \%$ estavam alinhados à esquerda, 17,8\% centralizados e nenhum alinhado à direita ou justificado. Tal predominância do alinhamento à esquerda é positiva por facilitar a leitura ao seguir a forma natural da escrita, respeitando o fluxo orgânico da linguagem (Bringhurst, 2005), e ao manter um espaçamento regular, o que evita a formação de "rios" ao longo do texto (Baines \& Haslam, 2002).

Apenas um dos conjuntos de textos observados utiliza hifenização - a saber, os blocos de texto de notícias da ETH Zurich. De fato, a hifenização deve ser evitada por dificultar a fluidez da leitura e por gerar impedimentos em termos de acessibilidade, ao impelir o leitor a suspender a compreensão do conteúdo entre o final de uma linha e o início da linha seguinte (Ferreira, 2019, p. 62). 
Quanto ao emprego de serifas, observando-se apenas os longos blocos de texto de notícias, constatou-se que $60 \%$ utilizam fontes sans serif. Já em leads e títulos, as fontes sem serifa são ainda mais predominantes: nos leads, representam $86,7 \%$, ao passo que em títulos representam $66,7 \%$.

Se, por um lado, estas estatísticas contrariam a constatação de Constantin (2013) de que os tipos serifados vêm dominando os textos de notícias; por outro, confirma-se a primazia dos tipos sem serifa na internet como um todo - isto é, não apenas em blocos de textos longos -, conforme a afirmação de Unger (2018, p. 171) de que as fontes sem serifa ainda são o "tipo dominante na web".

Tal preferência pelas sans serifs teve origem nos primórdios da reprodução de tipos em tela. As baixas resoluções, que frequentemente impediam que as serifas fossem apresentadas de forma satisfatória, transformavam-nas em elementos confusos e de distração. Mesmo que atualmente, graças às altas resoluções, as serifas já possam ser reproduzidas adequadamente em tela, os sans serifs continuam a ser amplamente empregados. Segundo Unger (2018, p. 171), seria por hábito que os designers gráficos tendem a reservar as fontes serifadas para aqueles textos mais longos que serão impressos no papel, e as fontes sem serifa para a web.

Talvez não à toa, dentre os 45 conjuntos de texto analisados no presente artigo, as duas fontes empregadas com maior frequência sejam as sans serifs Helvetica $(24,4 \%)$ e Arial $(13,3 \%)$.

A fonte Arial, por exemplo, é uma das mais usadas tanto na web quanto em impressos, apesar de mostrar-se, segundo algumas pesquisas, inadequada para a alfabetização de crianças. Isto se deveria em parte a aberturas pequenas em letras como 'a', 'e', ' $g$ ' e 's' (Unger, 2018, p. 171).

"Mas isso dificilmente parece afetar a aceitação da Arial como fonte padrão ou como fonte auto selecionada para muitos usuários - certamente não é ilegível e deve estar em algum lugar perto do centro da nuvem de tipos [...] por causa de seu uso frequente." (Unger, 2018, p. 171, tradução nossa)

Segundo Farias (2013, p. 105), diversas pesquisas concluíram que a legibilidade de fontes específicas dependeria em grande parte da familiaridade e de valores estéticos. Leitores tendem a ler melhor aquilo que leem com mais frequência (Gill, 1931; Licko, 1990, p. 15; Unger, 2016). A legibilidade, por conseguinte, corresponderia "simplesmente àquilo a que estamos acostumados" (Gill, 1931, p. 44):

"A neutralidade é alcançada quando algo é tão comum que desaparece da cognição normal. Portanto, a neutralidade é subjetiva e baseada no grau de experiência do observador. Então, quando dizemos 'projete uma fonte neutra', o que realmente queremos dizer é 'defina uma fonte que seja tão familiar na forma que nada nela será visivelmente novo'. Legibilidade é neutralidade. As fontes mais populares são as mais fáceis de ler; a popularidade as fez desaparecer da nossa cognição consciente. Depois de um certo tempo, é impossivel dizer se elas são fáceis de ler porque são comumente usadas ou se são comumente usadas porque são fáceis de ler." (Licko, 1990, p. 15, tradução nossa) 
Retomando a questão da preferência por fontes com ou sem serifa, aqui vale também mencionar uma diferença histórica entre ambas. No decorrer da evolução do design de tipos, Bigelow e Day (1983, p. 109-115) identificam dois estágios que se sucedem após a introdução de cada nova tecnologia. Primeiro, há um período de imitação, no qual as formas de letras proeminentes da geração tipográfica anterior servem como modelo para os novos desenhos. Em segundo lugar, "à medida que os designers se tornam mais confiantes e familiarizados com o novo meio, surgem projetos inovadores que não são meramente imitativos, mas exploram as forças e as limitações do meio" (Bigelow \& Day, 1983, p. 110, tradução nossa).

No caso da introdução da prensa com tipos móveis, no século $X V$, as primeiras letras glípticas ${ }^{1}$ imitavam manuscritos antigos e entalhes em pedra, o que incluía o uso de serifas. Foi somente no século $\mathrm{XIX}^{2}$ que os designers de tipos intensificaram a fase de experimentação $\mathrm{e}$ inovação, simplificando as formas das letras e eliminando as serifas (Clark, 2015, p. 16). Por consequência, apesar de fontes sans serif haverem surgido há mais de 200 anos, estas continuam sendo classificadas como modernas e universais, ao passo que as letras serifadas são associadas a instituições clássicas e tradicionais.

No que diz respeito aos resultados do presente levantamento, nota-se que universidades mais antigas - como a University of Oxford, a mais antiga do mundo de fala inglesa, inaugurada em 1096 - costumam enfatizar a questão da tradição em seus discursos (Oxford, 2021). Este tradicionalismo também se reflete no desenho de seus logos.

Com o intuito de visualizar uma possível relação entre o tempo de existência das instituições e o tipo de fonte utilizada em seus respectivos logos, no gráfico da Figura 2, comparamos o ano de inauguração de cada uma das 15 universidades examinadas e a classificação das fontes de seus logos atuais, isto é, com ou sem serifa.

Como referência, também indicamos no gráfico o ano comumente atribuído à invenção da prensa de tipos móveis (1450) e o ano de introdução dos tipos sans serif (1816). Observamos que, dentre as instituições em análise, todas aquelas cujo ano de inauguração é anterior a 1816, empregam fontes serifadas em seus logos atuais. Por conseguinte, as universidades que possuem logos com letras sem serifas foram todas inauguradas após 1816.

\footnotetext{
${ }^{1}$ O termo glíptico refere-se a "gravação, incisão e entalhe em metais, geralmente de diminutas dimensões" (Trevisan, 2015). Bigelow e Day (1983, p. 108-109) incluem naquilo que denominam de "processo glíptico" tanto o corte de punção manual quanto o mecânico. Mas Southall (1985) discorda da inclusão dos processos mecanizados nesta categoria, apontando que em cada estágio do processo, desde o desenho da letra até o corte da punção, o operador estaria traçando o contorno de uma forma ao invés de esculpindo um objeto: "Por falta de uma palavra melhor, eu descreveria o processo de corte mecânico como delineamento" (Southall, 1985, p. 16, grifo do autor, tradução nossa).

${ }^{2}$ Segundo Kane (2012, p. 36), as fontes sem serifa foram apresentados pela primeira vez por William Caslon IV em 1816. Destinadas principalmente ao uso em títulos, foram inicialmente chamadas de "grotescas" ou "gothic". Foi somente em 1832 que o fundidor inglês Vincent Figgins as classificou como "sans syrruf".
} 
Figura 2: Comparação entre o ano de inauguração das universidades e as fontes utilizadas em seus logos atuais. (Fonte: elaboração nossa, 2021)

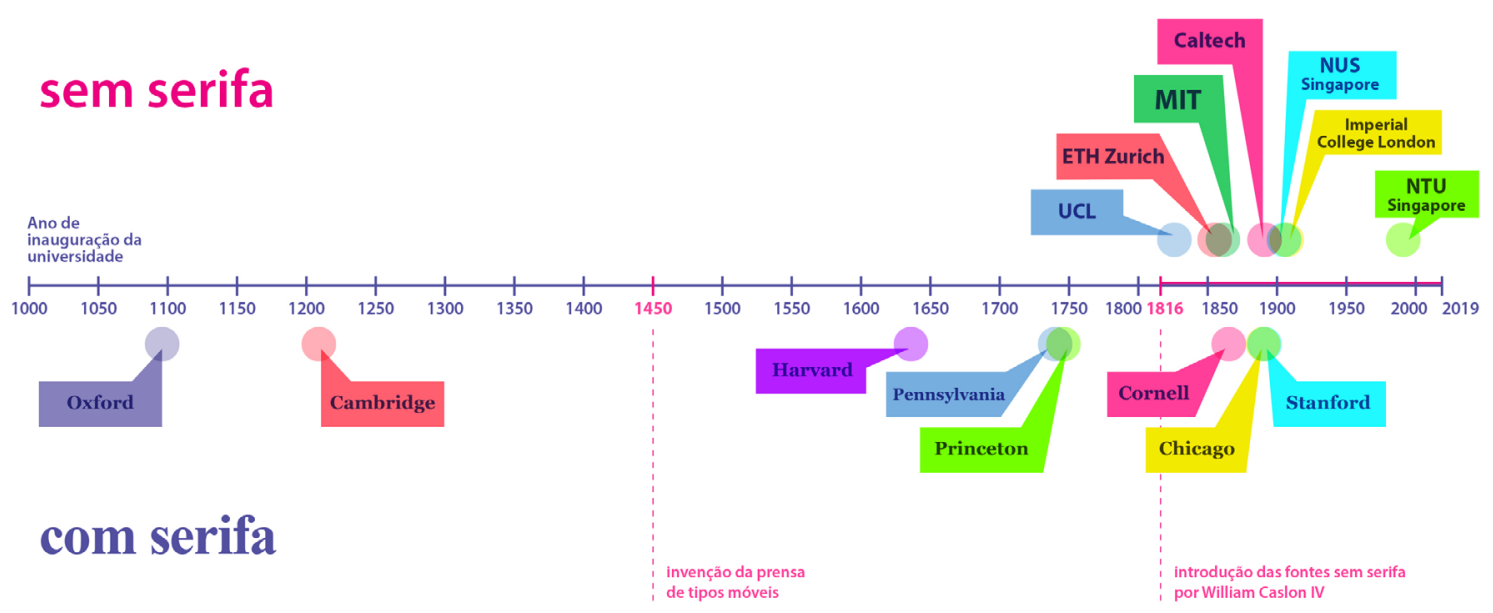

Quanto aos regionalismos, na América do Norte - região com o maior número de universidades no ranking - os logos com serifa predominam, somando $75 \%$ do total; ao contrário da Ásia, onde as fontes sans serif compõem 100\% dos logos. Na Europa houve maior equilíbrio: $60 \%$ dos logos sem serifa e $40 \%$ com.

Apesar de parecer haver um cuidado criterioso na escolha das fontes empregadas nos sites das universidades, dentre as 15 instituições observadas, apenas duas utilizavam fontes customizadas. Entende-se aqui "customizada" como uma fonte tipográfica personalizada, criada exclusivamente para uma empresa ou organização, de forma a "carregar e transmitir a identidade de uma instituição, até mesmo nas situações em que a própria marca está ausente, assumindo o papel de transportar, juntamente com o conteúdo da mensagem, valores, comportamentos e personalidade" (Cardinali, 2015, p. 9).

Uma das duas únicas fontes customizadas que foram identificadas no presente levantamento é a Stanford, fonte desenvolvida para a Stanford University. No site da instituição, no entanto, a fonte foi empregada apenas nas reproduções do logotipo da universidade; não sendo utilizada em blocos de texto ou títulos de notícias, por exemplo.

Já a outra fonte customizada, não apenas é vastamente empregada em todo o site da respectiva universidade, como também apresenta estreitas relações com a própria história da instituição. $O$ desenho da Princeton Monticello foi baseado em uma fonte criada por uma type foundry fundada na Philadelphia em 1796 por Archibald Binny e James Ronaldson. Os vínculos desta fonte tipográfica com a Princeton University datam da década de 1940, quando os tipos foram redesenhados pela Mergenthaler Company em colaboração com a editora da universidade. Foi também nessa época que a fonte passou a ser chamada Monticello. A partir dos anos 1980 foram desenvolvidas duas versões digitais da fonte, que foi finalmente reinterpretada em 2003 por Matthew Carter - também em parceria com a Princeton University Press (Creesy, 2006). 
A ubiquidade da fonte nas interfaces de comunicação da universidade e sua ligação com a identidade da instituição é tão marcante que Eric Li, vencedor da edição de 2018 do prêmio Jim Seawright Award, concedido pela Princeton University a trabalhos de artes visuais, criou uma nova fonte tipográfica apenas removendo as serifas da Princeton Monticello (Figura 3). Sobre sua fonte, a Monti Sans, ele escreve: "Estas novas formas, limpas de sua bagagem histórica [...], trazem nova luz a essa fonte centenária. [...] Monticello foi o passado. Monti Sans é o agora e o futuro" (Li, 2018, p. 4-5).

Figura 3: Monti Sans, fonte criada por Eric Li, aluno da Princeton University, ao remover as serifas da Princeton Monticello, tipografia oficial da universidade. (Fonte: Li, 2018)

\section{This}

\section{font}

\section{was}

handmade

by

literally

chopping

off

\section{the \\ serifs \\ of \\ Princeton \\ Monticello}

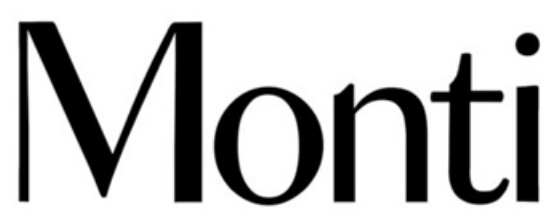

Sans

\section{Conclusões}

A partir dos dados coletados das 15 universidades examinadas para a presente pesquisa, ainda seria possivel desenvolver diversas outras discussões, mas nesta breve análise já foi possível inferir algumas conclusões. 
Os sites universitários têm acompanhado as tendências tipográficas em termos de corpo tamanho dos tipos -, de entrelinha, de alinhamento e hifenização. Mas, contrariando os resultados de Constantin (2013) para sites jornalísticos, no presente corpus, as fontes de texto de notícias ainda são predominantemente sem serifa.

No que diz respeito ao uso de cores, também constatamos uma conformidade com os grandes portais jornalísticos. Nas páginas internas de notícias dos sites analisados, em $96,67 \%$ dos casos utilizam-se tons escuros para os textos, e tons claros para os fundos. Mas apenas $26,67 \%$ da amostragem emprega as duas cores de contraste máximo, isto é, texto preto ( $\# 000)$ sobre fundo branco (\#fff). No geral, percebe-se uma preferência por contrastes mais amenos, como caracteres em cinza escuro sobre fundos beges.

Quanto aos logos das universidades, as serifas são ligeiramente prevalentes, representando $53,33 \%$ do total. Destacamos que $100 \%$ das instituições mais antigas, inauguradas anteriormente à introdução das fontes sans serif em 1816, mantêm mesmo em seus logos atuais o emprego de fontes com serifa.

Outro ponto relevante foi a identificação de uma fonte - a Princeton Monticello - cuja presença e influência permeia tanto a história da universidade quanto sua identidade atual, fazendo-se presente em diversas de suas mídias de comunicação, incluindo o site.

Percebe-se aí a influência da tipografia não apenas na estética e legibilidade dos textos de um site universitário, mas também no estabelecimento dos valores e narrativa da própria instituição.

Acreditamos que o presente artigo, ao levantar as práticas tipográficas atuais entre páginas de instituições de ensino superior, contribui com o delineamento de parâmetros objetivos para o desenvolvimento de produtos eficientes em termos de legibilidade e de transmissão da identidade das instituições que representam, enriquecendo o desenvolvimento de produtos similares aos analisados e representando contribuição metodológica ao levantamento de dados em tipografia.

\section{Referências}

Baines, P. \& Haslam, A. (2002). Type \& Typography. London: Laurence King.

Bigelow, C., \& Day, D. (1983). Digital Typography. Scientific American, 249(2), 106-119.

Bringhurst, R. (2005). Elementos do estilo tipográfico. São Paulo, Cosac Naify.

Callaghan, C. O. (2017). Comparing World University Rankings: THE, QS and Shanghai. Top Universities. Disponível em: www.topuniversities.com/student-info/universitynews/comparing-world-university-rankings-qs-shanghai

Cardinali, L. (2015). A tipografia customizada como elemento identitário em sistemas de identidades visuais. Dissertação de mestrado, Universidade de São Paulo, Brasil.

Compton, Y. S. Texto na tela. In: Lupton, E. (Org.). Tipos na tela (pp. 48-77) (M. Bandarra, Trad.). São Paulo: Gustavo Gili.

Constantin, J. (2013). Typographic Design Patterns and Current Practices 2013 Edition. Smashing Magazine. Disponível em: www.smashingmagazine.com/2013/05/typographicdesign-patterns-practices-case-study-2013 
Constantin, J. (2009). Typographic Design Patterns and Best Practices. Smashing Magazine. Disponível em: www.smashingmagazine.com/2009/08/typographic-design-survey-bestpractices-from-the-best-blogs

Creesy, C. (2006). Monticello: The History of a Typeface. Printing History: The Journal of the American Printing History Association. XXV(1).

Farias, P. L. (2016). Estudos sobre tipografia: letras, memória gráfica e paisagens tipográficas. Tese de Livre-docência, Universidade de São Paulo, Brasil.

Farias, P. L. (2013). Tipografia Digital: O impacto das novas tecnologias (4ª ed.). São Paulo: 2AB.

Ferreira, D. (2019). Design Editorial Algorítmico. Dissertação de Mestrado, Universidade de Coimbra, Portugal.

Gill, E. (1931). An Essay on Typography. Boston: David R. Godine.

Kane, J. (2012). Manual dos Tipos (R. Bettoni, Trad.). Barcelona: Gustavo Gili.

Li, E. (2018). Monti Sans. Princeton: Princeton University.

Licko, Z. (1990). Can Fine Typography Exist in the '90s? U\&/c, 17(3), 14-15. Disponível em: https://issuu.com/csisson/docs/u_lc_vol.17-3

Mozina, K., Podlesek, A. \& Bračko, S. (2019). Preserving typographic cultural heritage using contemporary digital technology. Journal of Cultural Heritage, 36, 166 - 173.

Oxford. (2021). Introduction and history. University of Oxford. Disponível em: https://www.ox.ac.uk/about/organisation/history

Princeton. (2021). Logo \& Graphic Identity - Office of Communications. Princeton University. Disponível em: https://communications.princeton.edu/guides-tools/logo-graphic-identity

QS. (2019). QS World University Rankings $® 2020$. Disponível em: www.topuniversities.com/university-rankings/world-university-rankings/2020

Sorkin, E. (2011). Why and how I am making Merriweather. Disponível em: https://ebensorkin.wordpress.com/2011/02/11/why-and-how-i-am-making-merriweather

Southall, R. (1985). Designing New Typefaces with Metafont. Stanford: Stanford University.

Spiekermann, E. \& Ginger, E. M. (1993). Stop Stealing Sheep \& find out how type works. San Francisco: Adobe.

Trevisan, R. (Ed.) (2015). Michaelis Dicionário Brasileiro da Língua Portuguesa. São Paulo: Editora Melhoramentos.

Unger, G. (2016). Enquanto você lê. (M. Galvão, Trad.). Brasília: Estereográfica.

Unger, G. (2018). Theory of Type Design. Rotterdam: nai010 Publishers.

Zirulnick, A. (2010). New world university ranking puts Harvard back on top. The Christian Science Monitor. Disponível em: https://www.csmonitor.com/World/2010/0916/New-worlduniversity-ranking-puts-Harvard-back-on-top

\section{Sobre as autoras}

Susana Narimatsu Sato, Mestre, USP, Brasil, snsato@usp.br

Clice de Toledo Sanjar Mazzilli, Profa. Dra., USP, Brasil, clice@usp.br 